\title{
Mechanics of Electrical Transmission Line Robot Inspector: Pendulum as a Dynamic Vibration Absorber
}

\author{
Bahrami Mohammad Reza ${ }^{1, *}$ \\ ${ }^{1}$ Innopolis University, 420500 Innopolis, Russia
}

\begin{abstract}
The purpose of this article is to design a new dynamic vibration absorber (DVA) and create a mathematical model to analyze the electrical transmission lines inspection robot movement on the line. First, the vibration of the electrical line while the robot inspector moves along it has been modeled considering the conductor as a stretched string and the robot as a moving load. It follows by using a double pendulum as the dynamic vibration absorber. To better understanding the performance of the DVA, the results are compared with the model in which a robot inspector is considered as a pendulum with a suspension base (here the conductor). This example shows the possibility of usage of the double pendulum as the DVA in electrical transmission inspection robots.
\end{abstract}

\section{Introduction}

Electric power transmission lines worldwide are used to transmit electricity generated in power plants to costumers. There exist underground transmission systems that are more desirable for the environment but expensive in comparison with overhead lines. Overhead power transmission lines although are cheap but are easily subject to environmental effects (natural weather). Long term subject of electrical transmission lines to the natural weather may cause stresses, material corrosion, etc. Regular expansion and contraction of power lines as the result of temperature changes cause fatigue and aging of materials which leads to fracture or deformation along the lines or may cause hot spots (power lost during the transmission). Therefore, it is required to inspect electrical transmission lines regularly.

Power companies use different methods to inspect electrical transmission lines according to the regional regulations. Typical inspections are ground inspection and aerial inspection using a helicopter. The efficiency of conventional methods highly depends on the experience of one technician who, through binoculars, covers the transmission lines (equipment as well).

There exists another type of inspections including unmanned aerial vehicles, robot inspectors, etc. Robot inspectors are intelligent, semi or fully autonomous devices utilizing different technics and technologies to check the status of electrical transmission equipment.

Different concepts for electrical transmission line robot inspectors are proposed and constructed [1-9]. One of the proposed structures is shown in Fig.1 [9]. The main idea of using such robots is to reduce the presence of humans in high-risk environments and have more precise results. These concepts mostly focus on bypassing obstacles.

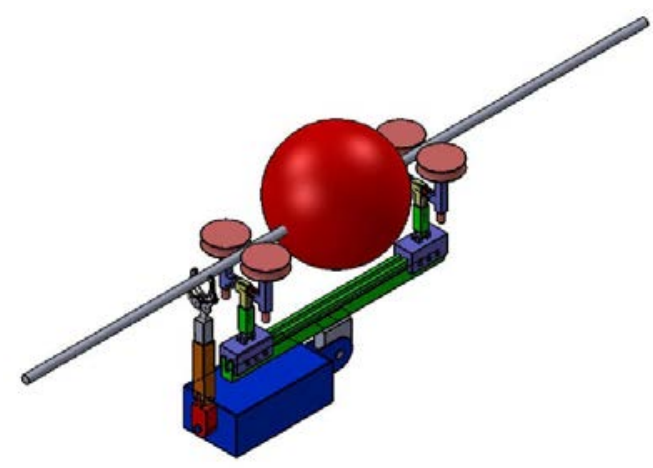

Fig. 1. Schematic of an electrical transmission line robot inspector [9].

Electrical line inspection robot structures are varied designing for different tasks [1-6]. As mentioned, the basic idea behind these concepts is bypassing the obstacles (electrical transmission line components) using different mechanisms. As experience and research shown in [2-8] very intensive fluctuations appear by the movement of the robot along the line. These vibrations occur even with the slow and steady motion of the diagnostic machine [10-12]. Since these fluctuations may cause inertia loads on the robot structure and may cause parametric oscillation in the vertical plane of the motion, designing a system to reduce the amplitude of vibration or even prevent is necessary [13-15].

The purpose of this article is to design a new dynamic vibration absorber (DVA) and create a mathematical model to analyze the electrical

\footnotetext{
* Corresponding author: mo.bahrami@innopolis.ru
} 
transmission lines inspection robot movement on the line. The article organized as follows: vibration of the electrical line while the robot inspector moves along it has been modeled considering the conductor as a stretched string and the robot as a moving load. It follows by using a double pendulum as the dynamic vibration absorber. To study the performance of the DVA, the results are compared with the model in which a robot inspector is considered as a pendulum with a suspension base (here the conductor).

\section{Vibration of the conductor due to the movement of the robot inspector on it}

The operation and performance of the robot inspector depend on many factors such as vibration. In this section, we study the vibration of the line conductor due to the motion of the robot inspector along the line in the horizontal and vertical plane of motion.

\subsection{Vibration in the horizontal plane of motion}

To study the dynamics of the conductor with a moving robot inspector we use the simplest model where the conductor is considered as a stretched string, and the robot inspector is considered as a moving load (Fig. 2).



Fig. 2. Stretched string with a moving object.

The equation for string deflection, $u(x, t)$, is known [16-20]:

$$
T u^{\prime \prime}+f(x, t)=\rho \ddot{u} .
$$

In (1) the string tension force is designated by $T$, the linear load (per unit length) is represented by $f$, and $\rho$ is the density. Initial conditions are $x=0, l: u=0$, $t=0: u=\dot{u}=0$, where $l$ is the string length. Prime and dot mean differentiation by coordinate $x$ and time $t$.

The linear load per unit length $f$ can be determined using the point $F(t)$, and delta function at a point where $x=\xi$ as

$$
f(x, t)=F(t) \delta(x-\xi(t)) .
$$

The point load moves through the line according to an arbitrary law $\xi(t)$ with the condition of $0<t<t_{f}, \xi(0)=0, \xi\left(t_{f}\right)=l$.

With $F(t)=$ constant and $\xi=v t$ (the load moves at a constant velocity $v$ ) we have deflection from [12]:

$$
\begin{aligned}
& u_{n}=\frac{F}{\lambda_{n}\left(v^{2}-c^{2}\right)} \sqrt{\frac{2}{T \rho l}}\left(v \sin \lambda_{n} c t-c \sin \lambda_{n} v t\right), \\
& \lambda_{n}=n \pi / l, \\
& c=\sqrt{T / \rho} .
\end{aligned}
$$

where $n$ is the number of members of the series.

Eq. (3) is solved by a mathematical computer program. The deflection and acceleration of the conductor have been shown in Fig. 3. The following parameters are used for calculation: $T=10 \mathrm{kN}, F=1 \mathrm{kN}$, $\nu=2 \mathrm{~m} / \mathrm{s}, \rho=5 \mathrm{~kg} / \mathrm{m}, l=200 \mathrm{~m}, \mathrm{n}=500$. Saw-tooth type vibration appears in the horizontal plane of motion although the motion of the robot inspector is steady (Fig. 3(a). Fast varying acceleration may cause inertial load on the robot showed in Fig. 3(b) and may cause parametric oscillations in the vertical plane of motion.

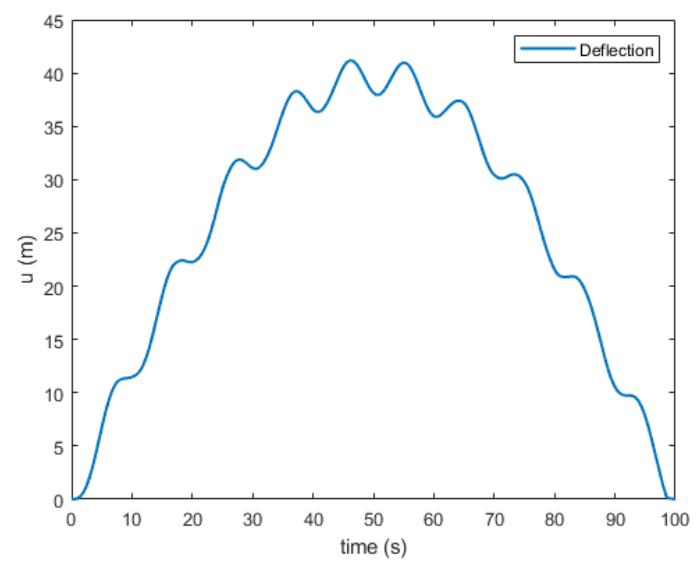

(a)

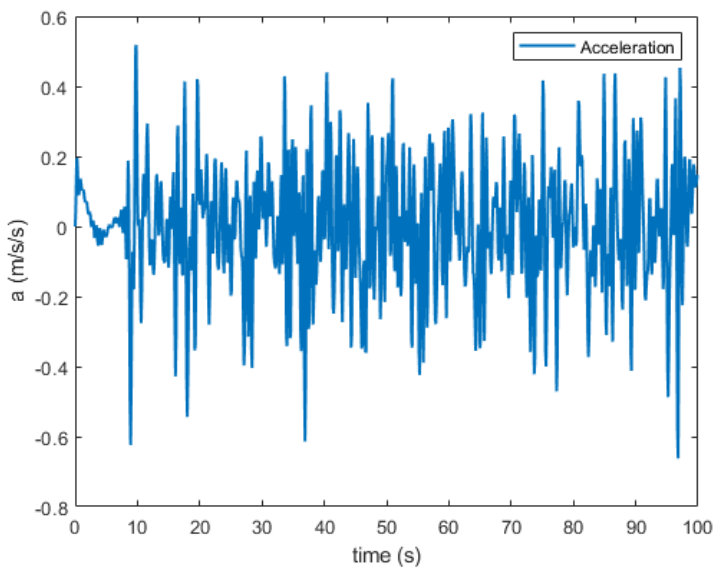

(b)

Fig. 3. (a) Deflection and (b) acceleration of the wire.

\subsection{Parametric oscillation in the vertical plane of motion}

The effect of saw-tooth vibration showed in Fig. 3(a), and parametric oscillations in vertical plane studied in [12]. In this model, the robot is considered as a pendulum with a movable suspension base (conductor). It is not the classical parametric resonance, but the increase of oscillation amplitudes can be observed limited by time. 
In this paper, we study pendulum with a movable suspension base (Fig. 4(a)) [12] to a better understanding of the performance of DVA while the inspection robot moves along transmission lines. The equation of motion of a pendulum with a movable suspension has been used:

$$
I \ddot{\theta}+(g-a(t)) r \theta=0 .
$$

The numerical solution of (4) obtaining by a mathematical computer program has been shown in Fig. 5(a) using the following parameters: $M=100 \mathrm{~kg}, r=0.3 \mathrm{~m}$, $g=9.98 \mathrm{~m} / \mathrm{s}^{2}$ with the initial conditions $\theta(0)=0.1, \dot{\theta}(0)=0 . a(t)$ is as shown in Fig. 3(a).

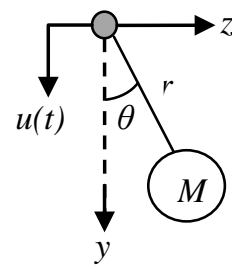

(a)

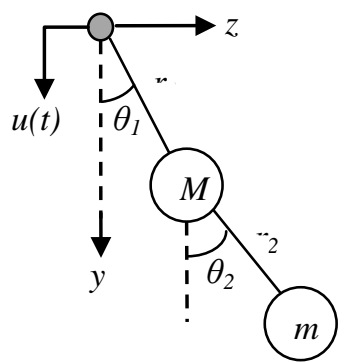

(b)
Fig. 4. Pendulum with suspension base (a) without DVA, (b) with DVA.

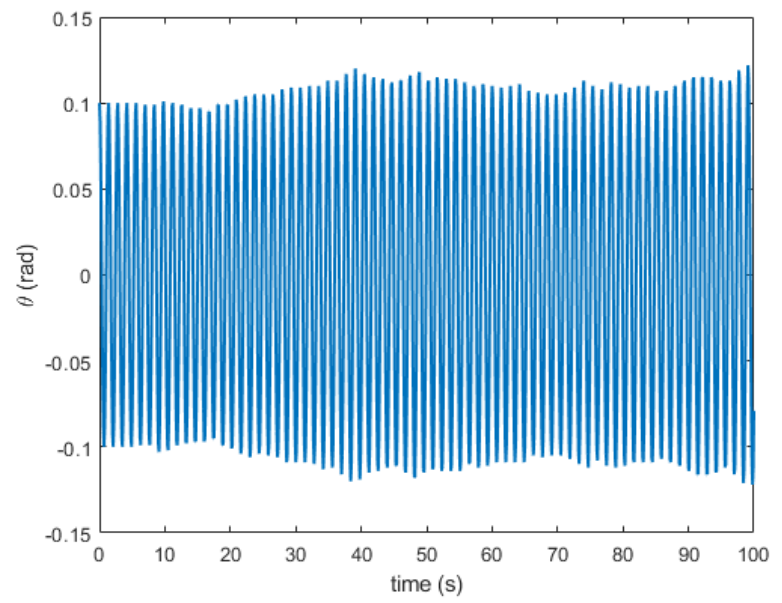

Fig. 5. Parametric oscillation of the pendulum with suspension base.

As shown in Fig. 5, one can see the increase in the amplitude of fluctuations as the time passes. This example shows the probability of the appearance of parametric vibrations in the vertical plane of the motion.

In this paper, to prevent dangerous oscillation in the vertical plane, dynamic vibration absorber (DVA) using a double pendulum with spring and damper in the second joint has been designed. The performance of the new DVA used in the inspection robot while traveling along the overhead transmission lines has been studied.

\section{Pendulum as a dynamic vibration absorber}

The concept of the dynamic vibration absorber DVA is based on the double pendulum to decrease the sway motion of the pendulum with a suspension base (Fig. 4(b)). In Fig. 4(b) $M$ is the robot mass or the pendulum mass, the deflection angles of first and second pendulums are designated by $\theta_{1}$ and $\theta_{2}$ measured in the $z-y$ plane from the vertical axis. The position of the center of mass of the pendulum is indicated by $r_{1}$. The tuned mass (second pendulum) $\mathrm{m}$ locates in the static position is shown by $r_{2} . k$ and $b$ are the spring stiffness and the damping coefficient of the DVA system located in the second joint. Function $u(t)$, deflection of the conductor obtained in section 2, describes the base motion in the vertical direction.

Considering the coordinate system as shown in Fig. 4, the positions and the velocities of the main system mass $\left(z_{1}, y_{1}\right)$ and the DVA mass $\left(z_{2}, y_{2}\right)$ are:

$$
\begin{aligned}
& \mathrm{z}_{1}=r_{1} \sin \left(\theta_{1}\right), \\
& \mathrm{y}_{1}=r_{1} \cos \left(\theta_{1}\right)+u(\mathrm{t}), \\
& \mathrm{z}_{2}=z_{1}+r_{2} \sin \left(\theta_{2}\right), \\
& \mathrm{y}_{2}=\mathrm{y}_{1}+r_{2} \cos \left(\theta_{2}\right), \\
& \dot{z}_{1}=r_{1} \dot{\theta}_{1} \cos \left(\theta_{1}\right), \\
& \dot{y}_{1}=-r_{1} \dot{\theta}_{1} \sin \left(\theta_{1}\right)+\dot{u}(\mathrm{t}), \\
& \dot{\mathrm{z}}_{2}=\dot{z}_{1}+r_{2} \dot{\theta}_{2} \cos \left(\theta_{2}\right), \\
& \dot{y}_{2}=\dot{y}_{1}-r_{2} \dot{\theta}_{\operatorname{\theta in}}(\theta) .
\end{aligned}
$$

One can write twice the kinetic energy using (5) as:

$$
\begin{aligned}
& 2 K=M\left(\dot{z}_{1}^{2}+\dot{y}_{1}^{2}\right)+m\left(\dot{z}_{2}^{2}+\dot{y}_{2}^{2}\right) \Rightarrow \\
& 2 K=M\left(r_{1}^{2} \dot{\theta}_{1}^{2}+\dot{u}^{2}-2 r_{1} \dot{u} \dot{\theta}_{1} \sin \left(\theta_{1}\right)\right)+\cdots \\
& \cdots+m\left\{r_{1}^{2} \dot{\theta}_{1}^{2}+r_{2}^{2} \dot{\theta}_{2}^{2}+\dot{u}^{2}+\cdots\right. \\
& \cdots+2 \mathrm{r}_{1} \mathrm{r}_{2} \dot{\theta}_{1} \dot{\theta}_{2} \cos \left(\theta_{1}-\theta_{2}\right)-\cdots \\
& \left.\cdots-2 \mathrm{r}_{1} \dot{u} \dot{\theta}_{1} \sin \left(\theta_{1}\right)-2 \mathrm{r}_{2} \dot{u} \dot{\theta}_{2} \sin \left(\theta_{2}\right)\right\} .
\end{aligned}
$$

The potential energy is obtained by:

$$
\begin{aligned}
& U=\frac{1}{2} k\left(\theta_{2}-\theta_{1}\right)^{2}-m r_{2} g \cos \theta_{2}-\cdots \\
& \cdots-(M+m) g r_{1} \cos \theta_{1}
\end{aligned}
$$

General forces are:

$$
\begin{aligned}
& Q_{\theta_{1}}=b\left(\dot{\theta}_{2}-\dot{\theta}_{1}\right), \\
& Q_{\theta_{2}}=-b\left(\dot{\theta}_{2}-\dot{\theta}_{1}\right) .
\end{aligned}
$$

One can obtain the system of equations after completing all the necessary actions in accordance with the Lagrange equation (second-order), for general coordinates $\theta_{1}$ and $\theta_{2}$ : 


$$
\begin{aligned}
& (M+m) r_{1} \ddot{\theta}_{1}+m r_{2} \ddot{\theta}_{2} \cos \left(\theta_{1}-\theta_{2}\right)+\cdots \\
& \cdots+m r_{2} \dot{\theta}_{2}^{2} \sin \left(\theta_{1}-\theta_{2}\right)+b\left(\dot{\theta}_{1}-\dot{\theta}_{2}\right)+\cdots \\
& \cdots+k\left(\theta_{1}-\theta_{2}\right)+(M+m) \sin \left(\theta_{1}\right)(g-a(t))=0, \\
& r_{2} \ddot{\theta}_{2}+r_{1} \ddot{\theta}_{1} \cos \left(\theta_{1}-\theta_{2}\right)-r_{1} \dot{\theta}_{1}^{2} \sin \left(\theta_{1}-\theta_{2}\right)+\cdots \\
& \cdots-b\left(\dot{\theta}_{1}-\dot{\theta}_{2}\right)-k\left(\theta_{1}-\theta_{2}\right)+\cdots \\
& \cdots+\sin \left(\theta_{2}\right)(g-a(t))=0 .
\end{aligned}
$$

The numerical solution of (9) been obtained by setting $a(t)$ as shown in Fig. 3(b). Fig. 5 shows the result for two cases (a) when spring and damper on the second joint are not connected and (b) are connected. The following parameters are used: $M=100 \mathrm{~kg}, m=2 \mathrm{~kg}$, $r_{1}=0.3 \mathrm{~m}, r_{2}=0.5 \mathrm{~m}, k=0.1 \mathrm{~N} / \mathrm{m}, b=25 \mathrm{~N} . \mathrm{s} / \mathrm{m}$ with initial conditions $\theta_{1}(0)=0.1, \theta_{2}(0)=\dot{\theta}_{2}(0)=\dot{\theta}_{1}(0)=0$.

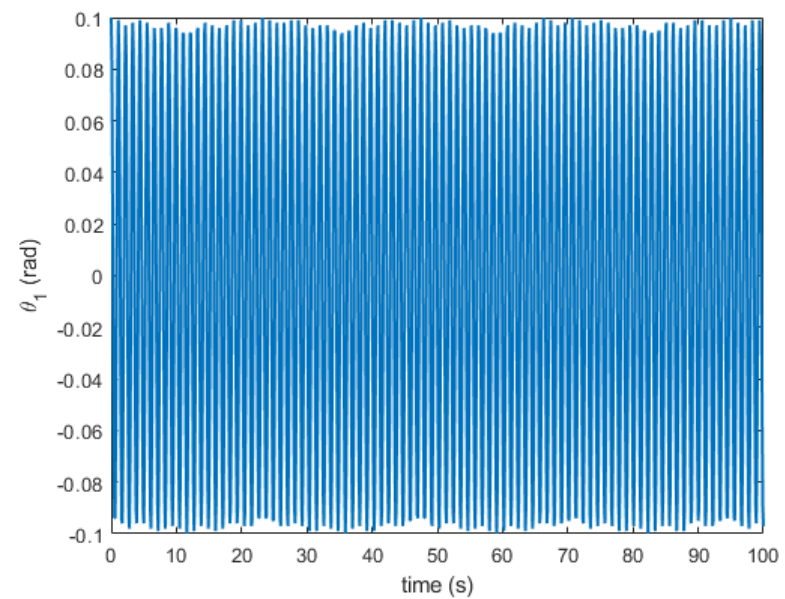

(a)

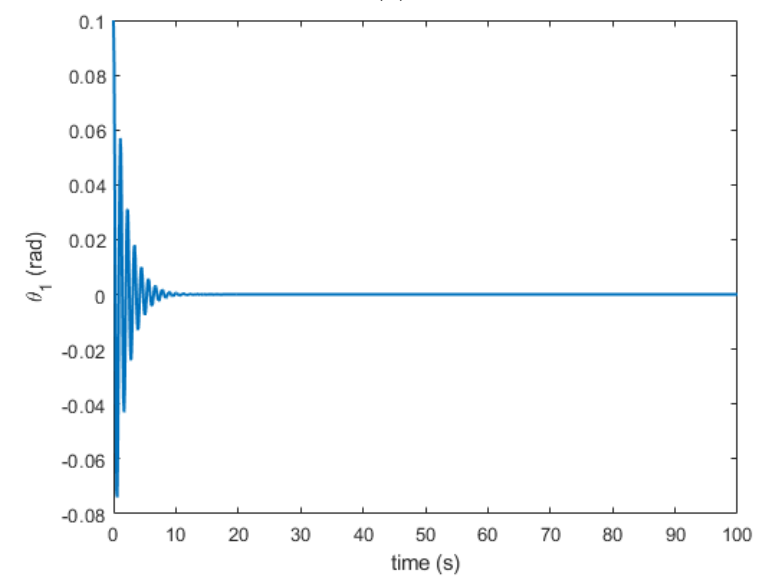

(b)

Fig. 5. Performance analysis of DVA: double pendulum (a) without and (b) with spring and damper in the second joint.

Fig. 5(a) shows the amplitude at least does not increase by the time of the process when spring and damper are not connected in the second joint. The amplitude of fluctuations decreased to zero by the time of the process as shown in Fig. 5(b).

This example shows the possibility of usage of the double pendulum as the DVA in electrical transmission inspection robots.

\section{Conclusion}

To study the dynamical behavior of the motion of inspection robot moving along the electrical transmission lines a mathematical model has been created. Vibration in the horizontal and vertical plane of motion has been investigated. As a result, intensive fluctuations observed in the horizontal plane which causes the parametric type oscillations in the vertical plane. To avoid dangerous oscillation in the vertical plane, a dynamic vibration absorber has been designed using double pendulum principles. The result shows the possibility of usage of DVA in electrical transmission inspection robots and it can be useful for engineers to select proper parameters for robot inspector.

\section{References}

1. M. Nayyerloo, W. Wang, et. al., J. Field Rob. (2009), ISBN: 978-953-307-001-8

2. K. Toussaint, N. Pouliot, S. Montambault, J. Field Rob, 26(5), 477-499 (2009)

3. S.I. Aoshima, T. Tsujimura, T. Yabuta InProceedings. IEEE/RSJ Int. Workshop IROS 89, 414-421 (1989)

4. J. Sawada, K. Kusumoto, Y, Maikawa, et al., A IEEE Trans. on P. Del. 6(1), 309-315 (1991)

5. M. Higuchi, Y. Maeda, S. Tsutani, S. Hagihara J. Rob. Soc. Japan, 9(4), 457-463 (1991)

6. T. Tsujimura, T. Morimitsu, Rob. \& auton. Sys. 20(1), 85-98 (1997)

7. S. Montambault, N. Pouliot, In 6th Int. Conf. FSR, 42 (2007)

8. P. Debenest, M. Guarnieri, K. Takita, et al., IEEE int. conf. on rob. and aut. (2008).

9. M.R. Bahrami Lect. Notes in Mech. Eng. : Adv. in Mech. Eng., Springer, 67-73 (2016)

10. V.V. Eliseev, M.R. Bahrami, St. Petersburg Polytech. Uni. J. 214, 200-207 (2015)

11. V.V. Eliseev, M.R. Bahrami, Moscow: Bulletin of eng. 6, 19-22 (2016)

12. M.R. Bahrami, In MATEC Web of Conf. 224 (2018 EDP Sciences.) https://doi.org/10.1051/matecconf/201822402021

13. M.R. Bahrami, S.A. Abed Vibroeng. PROCEDIA, 25 , 60-64

(2019) https://doi.org/10.21595/vp.2019.20807

14. M.R. Bahrami, S.A. Abed, IOP Conf. Series: Mat. Sci. \& Eng., (2019). (to be published)

15. M.R. Bahrami, Vibroeng. PROCEDIA (2020) (to be published)

16. V.V. Eliseev, Mechanics of Deformation of Rigid Body (St. Petersburg: Polytech. Uni. Pub., 2006)

17. A.N. Tikhonov, A.A. Samarsky, Equations of mathematical physics, (Moscow: Science, 1979)

18. D.R. Merkin Introduction to thread mechanics (Moscow: Science1981) 
19. V.A. Svetlitsky, Mechanics of flexible rods and threads (Moscow: Mech. Eng. 1978)

20. M.R. Bahrami Mathematical modeling of physical systems through bond graph (St. Petersburg:
Polytech. Uni. Pub., 2018) ISBN 978-5-7422-6186- 